\title{
Dental Bio-metrics as Human Personal Identifier using Pixel Neighborhood Segmentation Techniques
}

\author{
Sunita Sood, Ranju Kanwar, Malika Singh \\ ECE Dept., \\ Punjab College of Engineering and Technology
}

\begin{abstract}
Dental Biometrics has emerged as vital bio-metric information of human being on account of its stability and invariant nature and of course uniqueness. The presented work shows the dental unique feature set that may be used for claiming of the human personal identity using dental radiograph (x-ray graph).. The complete jaw is divided into four parts: left and right upper jaws, left and right lower jaws. Unique feature set includes number of teeth in each portion, maximum and minimum size in each portion, area and perimeter of each tooth in each portion and information regarding missing teeth in any of the portion. Later on, a database of features along with person under scanner profile is maintained for retrieval purposes.
\end{abstract}

\section{INTRODUCTION}

Dental Radiograph based human bio-metric information is the latest research area in the biometric identifier domain. The finger print and facial biometric suffers from the tampering problem as they are easy to manipulate. Even the face has the biggest threat of tampering. Face based biometric is not a reliable biometric as face is most susceptible to variation even by small change. However, dental profile is not possible to get tampered. No one would dare to get into it. As this being the costly affair and even the painful should be.

Dental biometrics should not only be the base of identification of a person but rather it should be treated as a confirmatory tool. For example, if a person's face is completely damaged and its identification is not [possible by face, then dental biometrics may used as confirmatory tool along with other like finger prints, vein impression etc.

However, the database generation using dental based bio-metrics features is not very common and needs a systematic arrangement as it involves the $\mathrm{x}$-ray device for getting the profile. X-ray machines are now-a-days digitized and a digital $x$-ray of the dental profile may be obtained in just short time. But, the problem is that the taking dental profile is not volunteer in nature. The person under scanner made to be present forcefully.

\section{BRIEF LITERATURE SURVEY}

From the literature study, it has been observed that the dental radio graphs are permanent forever and do not vary with age. Also, the dental graph is almost not possible to tamper. [1]

The dental radiograph suffers from the disadvantage that the person under scanner has to be enforced for acquiring its dental radio graph as the same is not voluntary like finger print or face based bio-metrics. [2].

The teeth segmentation is important part of the system. Normally the teeth are segmented using the pixel neighborhood connectivity based. The pixel neighborhood connectivity gives fair results for the dental radiographs. [3]
The image thresholding plays vital role before segmentation of the teeth for analysis. Otsu algorithm works fine when $\mathrm{x}$-ray images of teeth are under process. [4]

Feature vector set normally consists of statistical features and that do not vary when the size or zoom in out effect may have presence on images. [5]

Dental profile based biometrics is the most secure biometrics that remains safe and unchanged throughout the life and even after expiry of a person. The dental biometrics may be thought of to be fixed at the age of 7-8 years of age and then it becomes stable.

\section{ALGORITHM}

The dental radiograph based biometric system starts with image acquisition. Dental Image is acquired using X-Ray image Source. The $\mathrm{x}$-ray image is in gray color format and needs to be binarized in order to obtain the black and white image with white as background and teeth objects as black color.

The image thresholding is done using Otsu algorithm. Otsu algorithm works on the principal of minimum within class variance. Once the threshold $\mathrm{T}$ is selected. The image pixels with gray color intensity less than $\mathrm{T}$ are made black and those greater than $\mathrm{T}$ are made white. This gives the binary image with some noisy pixels in the form of salt and pepper noise.

Further, noise pixels are removed using salt and pepper algorithm. The pixels of either white or black color intensity lying alone are removed to get the noise free image. A dental image is shown below:

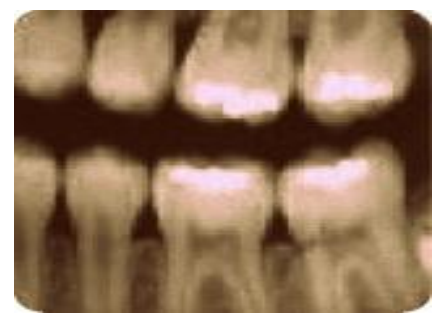

The binary image using the Otsu algorithm of the above is shown below:

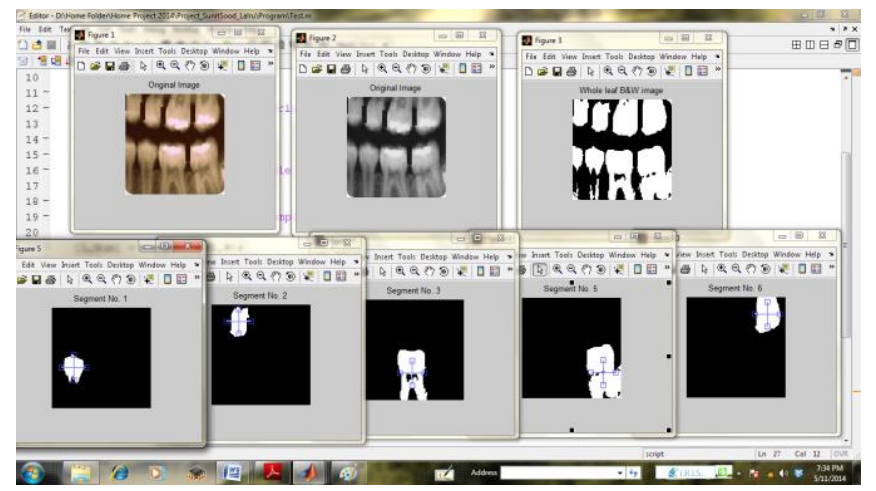


The binary image is now divided into four parts: top left, Top right, Bottom left and Bottom right. Each jaw has fixed no. of teeth and information about the missing teeth. Missing teeth can be found by analyzing the distance between the two teeth. If a tooth is missing between two teeth, then the distance between the same will be higher than the normal case.

All the feature parameters are normalized with respect to size i.e. mean radius. This gives the feature parameters independent of the size of the teeth that may vary on account of the distance of $\mathrm{x}$-ray $\mathrm{m} / \mathrm{c}$ from the jaw. Or the image may be zoomed in or out after getting the $\mathrm{x}$-ray.

The feature vector set is computed for each single tooth. For, the teeth are segmented using bwareaopen and bwlabel command in matlab. Both of the commands are based on 8-coonectivity of pixel neighborhood. The pixel's group is labeled based on their association within group and labels are assigned as number. Based on the labels, the teeth are segmented in single frame and feature vector set is computed.

An 8-connected pixel neighborhood is shown below:

\begin{tabular}{|l|l|l|}
\hline P8 & P1 & P2 \\
\hline P7 & P0 & P3 \\
\hline P6 & P5 & P4 \\
\hline
\end{tabular}

\section{EXPERIMENTS AND RESULTS}

The presented algorithm has been tested on a test image as shown below:

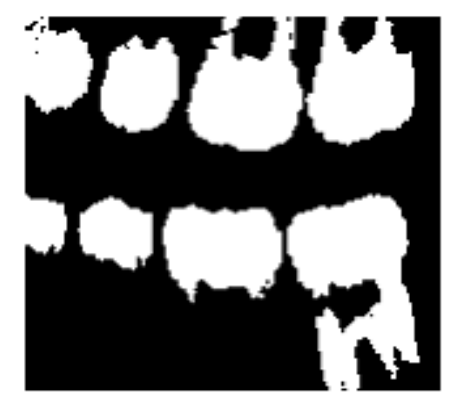

The teeth are counted in all four parts and summarized in as below:

$\begin{array}{ll}\text { No. of teeth in top left } & =2 \text {; } \\ \text { No. of teeth in top right } & =2 \text {; } \\ \text { No. of teeth in bottom left } & =2 \text {; }\end{array}$

No. of teeth in bottom right $=2$;

\begin{tabular}{|c|c|c|}
\hline Teeth No. & Area & Perimeter \\
\hline 1 & 213 & 100 \\
\hline 2 & 219 & 112 \\
\hline 3 & 256 & 134 \\
\hline 4 & 299 & 176 \\
\hline 5 & 110 & 78 \\
\hline 6 & 156 & 89 \\
\hline 7 & 239 & 122 \\
\hline 8 & 312 & 170 \\
\hline
\end{tabular}

The teeth shape may be estimated by computing the standard deviation of the teeth profile. A high value of standard deviation may suggest the breakage in tooth. For a regular shape tooth, the standard deviation should be as that of an elliptical shape. If the tooth is broken, then the standard deviation will increase.

\section{CONCLUSION}

The presented algorithm is designed using the matlab 7.5 version and is under testing phase. Further, the dental radiograph is not readily available for use. There is a dependency of the same on availability of $\mathrm{x}$-ray $\mathrm{m} / \mathrm{c}$ and even the person willingness. However, the algorithm has been tested on no. of radiographs and a correlation is established between the feature vector and the person bearing the same. The feature vector set has ability to identify the person from the data base if the feature vector set is used for search purposes.

\section{REFERENCES}

[1] Hong Chen, Student Member, IEEE, and Anil K. Jain, Fellow, IEEE, "Dental Biometrics: Alignment and Matching of Dental Radiographs", IEEE TRANSACTIONS ON PATTERN ANALYSIS AND MACHINE INTELLIGENCE, VOL. 27, NO. 8, AUGUST 2005

[2] Aparecido Nilceu Marana1, Elizabeth B. Barboza2, João Paulo Papa3,Michael Hofer4 and Denise Tostes Oliveira5, "Dental Biometrics for Human Identification" www.intechopen.com

[3] Shubhangi C. Dighe, Revati Shriram, "Dental Biometrics for Human Identification Based on Dental Work and Image Properties in Periapical Radiographs", Dept. Of Instrumentation and Control Cummins College of Engineering for Women, India

[4] Vijayakumari Pushparaj, Ulaganathan Gurunathan, and Banumathi Arumugam, "An Effective Dental Shape Extraction Algorithm Using Contour Information and Matching by Mahalanobis Distance", J Digit Imaging. Apr 2013; 26(2): 259-268. Published online Jun 14, 2012. doi: 10.1007/s10278-012-9492-4

[5] Amina Khatra, "DENTAL RADIOGRAPHS AS HUMAN BIOMETRIC IDENTIFIER: AN EIGEN VALUES/EIGEN VECTOR APPROACH", Cibtech Journal of Bio-Protocols ISSN: 2319-3840 (Online), 2013 Vol. 2 (3) SeptemberDecember, pp.6-9/Amina

[6] Shubhangi Dighe1, Revati Shriram2, "Preprocessing, Segmentation and Matching of Dental Radiographs used in Dental Biometrics" Volume 1, No.2, May - June 2012 International Journal of Science and Applied Information Technology.

[7] Shaishavkumar Parmar1 Mehul Amin2, "Determination of unique Feature set for AM Bitewing Dental Radiographs as a Bio-metric information", IJSRD - International Journal for Scientific Research \& Development| Vol. 1, Issue 10, 2013 ISSN (online): 2321-0613

[8] Nourdin Al-sherif, Ayman Abaza, and Hany Ammar "Dental Record Retrieval Based on Guided SIFT Descriptors", IICIT 2012

\section{Author's Profile}

Ms. Sunita received her B.Tech. in ECE from shahid udham singh College of Eng. \& Technology, Tangori and is pursuing her M.Tech. from Punjab college of Eng. \& Technology, lalru mandi,Mohali. Hers field of expertise is in image processing based application development 